\title{
Regional Flux Analysis of Longitudinal Atrophy in Alzheimer's Disease
}

\author{
Marco Lorenzi ${ }^{1,2}$, Nicholas Ayache ${ }^{1}$, and Xavier Pennec ${ }^{1}$ for the Alzheimer's \\ Disease Neuroimaging Initiative ${ }^{\star}$ \\ 1 Project Team Asclepios, INRIA Sophia Antipolis, France \\ 2 LENITEM, IRCCS San Giovanni di Dio, Fatebenefratelli, Italy
}

\begin{abstract}
The longitudinal analysis of the brain morphology in Alzheimer's disease( $\mathrm{AD})$ is fundamental for understanding and quantifying the dynamics of the pathology. This study provides a new measure of the brain longitudinal changes based on the Helmholtz decomposition of deformation fields. We used the scalar pressure map associated to the irrotational component in order to identify a consistent group-wise set of areas of maximal volume change. The atrophy was then quantified in these areas for each subject by the probabilistic integration of the flux of the longitudinal deformations across the boundaries. The presented framework unifies voxel-based and regional approaches, and robustly describes the longitudinal atrophy at group level as a spatial process governed by consistently defined regions. Our experiments showed that the resulting regional flux analysis is able to detect the differential atrophy patterns across populations, and leads to precise and statistically powered quantifications of the longitudinal changes in $\mathrm{AD}$, even in mild/premorbid cases.
\end{abstract}

\section{Introduction}

The longitudinal analysis of the brain morphology in Alzheimer's disease(AD) is fundamental for understanding and quantifying the dynamics of the pathology. The analysis of time series of MR images has been based on two different paradigms: hypothesis free and regional analysis. In the former case, the longitudinal atrophy is modeled at fine scales on the whole brain such as in the voxel/tensor based morphometry and cortical thickness analysis [1, 2]. These methods are useful for exploratory purposes, but usually lack robustness for a reliable quantification of the changes at the subject level, due to the high variability of the measurements and the multiple comparison problems. On the other hand, the regional analysis is focused on the detection of significant changes on

\footnotetext{
* Data used in preparation of this article were obtained from the Alzheimer's Disease Neuroimaging Initiative (ADNI) database (www.loni.ucla.edu/ADNI). As such, the investigators within the ADNI contributed to the design and implementation of ADNI and/or provided data but did not participate in analysis or writing of this report. A complete listing of ADNI investigators can be found at: www.Ioni.ucla.edu/ADNI/Collaboration/ADNI_Authorship_list.pdf
} 
regions which are usually identified thanks to segmentation. For instance, the boundary shift integral identifies the longitudinal atrophy as the shift of the segmented boundaries [3], and led to powered measure for the longitudinal hippocampal changes in Alzheimer [4. However, this kind of approaches relies on strong a priori hypotheses on the localization of the dynamics of interest, and might fail to detect more complex patterns of changes which are likely to underly the evolution of the pathology. Providing a longitudinal measure which could at the same time identify, consistently localize, and reliably quantify the longitudinal changes is crucial for understanding the dynamics of the pathological evolution and to provide stable measures for the clinical setting.

Non rigid registration encodes the morphological changes between pairs of longitudinal MRIs as deformation fields. It has been employed for both whole brain exploratory analysis and regional quantification, for instance through the Jacobian determinant analysis. However, the regional quantification still relies on prior segmentation, and is still sensitive to the biases, for instance for the numerical derivative required for computing the Jacobian. The deformation fields implicitly encode the spatial location of relevant atrophy processes, and novel analysis techniques are required to consistently extract and analyze these features. It has been proposed in [5] to parametrize the deformations by irrotational and divergence-free components, according to the Helmholtz decomposition of vector fields. If we assume that the atrophy can be completely described by a change of volume, then it is completely encoded by the irrotational part, while the divergence-free one only accounts for the tissue reorganization. Thus, the maximal/minimal locations of the irrotational potential define the centers of expanding and contracting regions, and may represent a promising measure for morphometric studies. A different measure of volume change associated to the deformation field is the flux across surfaces [6], which is the mathematical formulation of the boundary shift. However flux-based analysis has been seldom used in morphometric studies, due to the complexity of reliably integrate vector normals on probabilistic segmentations of the surface boundaries.

In this study we propose the regional flux analysis, a new approach for the study of morphological changes based on the Helmholtz decomposition of vector fields. In Section 1 we introduce the Helmholtz theorem, and the relationship between pressure and flux of deformations. These measure are used in Section 2 to consistently define through a hierarchical model the subspace of regions involved in the atrophy processes. These regions are then used at the subject level for the probabilistic flux integration. Finally, the framework is applied in Section 3 on a large sample of longitudinal observation from the ADNI dataset [7, to describe and quantify the pathological changes at different clinical stages, from premorbid, to early and late Alzheimer stages.

\section{Helmholtz Decomposition for Stationary Velocity Fields}

The present work is based on the registration based on stationary velocity fields (SVF), which has been already applied for the longitudinal analysis of 
deformations [8], and for which an implementation of the log-Demons algorithm is easily available 19 .

Pressure Potential and Flux through a Region. The Helmholtz theorem states that, given a vector field $v$ defined on $\mathbb{R}^{3}$ which vanishes when approaching to infinity, it can be uniquely factored as the sum of an irrotational and a divergence free component, $v=\nabla p+\nabla \times A$. The irrotational component $\nabla p$ is the gradient of a scalar pressure (potential) field $p$. Since $\nabla \times \nabla p=0$, the component encodes the information concerning the volume change. On the other hand the divergence-free component is by definition such that $\nabla \cdot \nabla \times A=0$ and therefore it describes the rotational part of the velocity. Finally, the flux of a stationary velocity field across a given surface $\partial V$ is given by the Divergence (or Ostrogradsky's) theorem, and can be rewritten as $\oint_{\partial V} v \cdot \boldsymbol{n} d S=\int_{V} \nabla \cdot v d V$. Recently the Helmholtz decomposition has been introduced in the Demons registration in order to estimate incompressible deformations [10]. Here we propose to use it on the contrary for the analysis of the compressible part, which encodes the observed matter loss as a smooth compression/expansion process. In such a model, the associated divergence quantifies the apparent anatomical changes as the flux of the estimated vector field across surfaces.

Topology of Pressure Fields. Theoretically, one could partition the whole space into critical areas of positive and negative divergence, each of them containing a critical point of local maximal/minimal pressure (Figure 1). From the divergence theorem, the flux across the boundaries of these areas is either flowing inward or outward. The saddle points for the pressure are on the boundaries of those regions, and identify a change in the flow.

The analysis of the critical points of a pressure map can be addressed by the Morse-Smale theory as a topological problem, leading to a complex of regions, boundaries, edges and vertices. Although intriguing, the application of such concepts to the medical imaging is still difficult, due to the missing statistical version of the Morse theory. In order to obtain a tractable approach to the problem, we propose to first focus on the definition of a consistent subset of critical regions across subjects, to robustly describe the atrophy processes at group level as a spatial process governed by key areas. This is a first step towards a topology definition and provides a sparse description of the deformation.

\section{Flux-Based Analysis of Longitudinal Trajectories}

The goal of this section is to estimate the group-wise set of critical regions, from the locations of maximal/minimal pressure. These regions are then used to evaluate the flux of the longitudinal deformations at the subject level.

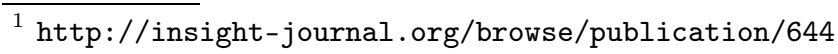




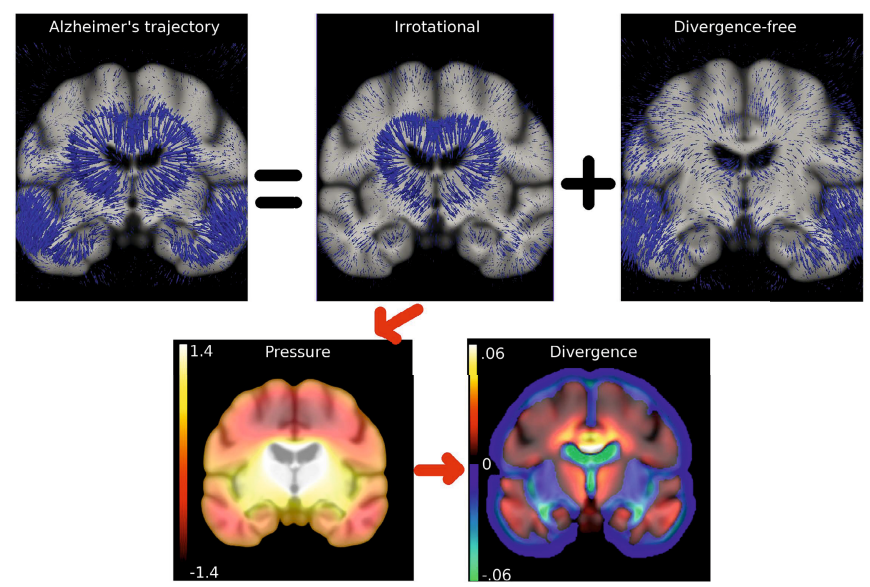

Fig. 1. Helmholtz decomposition of a longitudinal trajectory in Alzheimer's disease, and pressure potential and divergence maps associated to the irrotational component. The divergence describes the critical areas of local expansion and contraction.

Group-Wise Pressure Potential from Longitudinal SVFs. Consider the longitudinal observations from a group of subjects composed of baseline $I_{0}^{i}$ and follow-up $I_{1}^{i}$ brain scans. For each subject $i$, the log-Demons non rigid registration of the pair $I_{0}^{i}, I_{1}^{i}$ estimates the longitudinal trajectory of changes as a diffeomorphism parametrized by stationary velocity field $\exp \left(v_{i}\right)$, such that $I_{0}^{i} \circ \exp \left(v_{i}\right) \simeq I_{1}^{i}$. The SVF $v_{i}$ can then be decomposed according to the Helmholtz theorem in order to identify the corresponding pressure map $p_{i}$.

One interest in this decomposition is that the transport of each atrophy trajectory $\varphi_{i}=\exp \left(v_{i}\right)=\exp \left(\nabla p_{i}\right)$ through a subject-to-template deformation $\psi_{i}$ can be obtained by simple scalar interpolation of the pressure field $\varphi_{i}^{T}=\exp \left(v_{i}^{T}\right)=\exp \left(\nabla\left(p_{i} \circ \psi_{i}\right)\right)$, rather than parallel transporting vector quantities, $\varphi_{i}^{T}=\exp \left(\Pi^{\psi_{i}}\left(v_{i}\right)\right)$, which generally leads to computationally intensive and potentially more unstable operations.

The pressure maps in the template space $p_{i} \circ \psi_{i}$ are integral quantities, and might differ by an arbitrary constant. However, an average pressure map can still be consistently defined either as $\bar{p}=\overline{p_{i} \circ \psi}$, or as the pressure map $\bar{p}$ associated to $\bar{v}=\overline{v_{i}^{T}}=\overline{\nabla\left(p_{i} \circ \psi_{i}\right)} 2$

Probabilistic Estimation of Group-Wise Critical Regions. Let $\left\{x_{k}\right\}$ be the set of critical points, maxima and minima, of $\bar{p}$. These points define the critical areas $T_{k}$ of local expansion and contraction, i.e. of positive and negative divergence. Then, the probability of a point $x$ to belong to a critical region depends on the proximity to the region $T_{k}$, and on the observed divergence. We can express this through the Bayes rule:

\footnotetext{
$\overline{{ }^{2} \text { In fact, if } p_{i}^{\prime}}=p_{i} \circ \psi_{i}+c_{i}$, with $c_{i}$ constant, then $\bar{p}=\overline{p_{i} \circ \psi_{i}}+\bar{c}$ leads to $\bar{v}=\nabla \bar{p}=$ $\overline{\nabla\left(p_{i} \circ \psi_{i}\right)}$.
} 


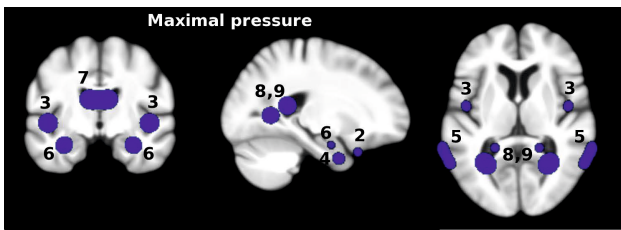

CSF areas surrounding:

E1 - inferior orbital cortex

E2/3 - insula/superior temporal pole

E4 - Mid-temporal pole

E5 - Sup/Mid temporal pole

Ventricles areas:

E6 - Temporal horn hippocampus

E7/8/9 - Ventricles

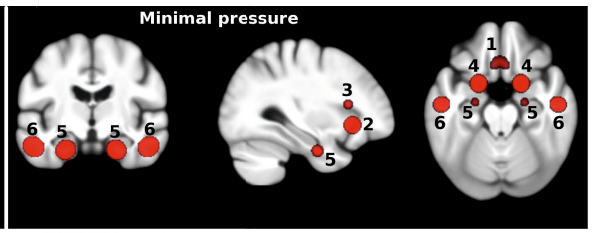

GM/WM regions:

C1 - Gyrus rectus

C2 - Insula

- Inferior frontal gyrus

- Amygdalae/parahipp gyrus

- Hippocampus

- Temporal poles

Fig. 2. Critical points associated to the Alzheimer's average pressure map

$$
P\left(x \in T_{k} \mid \nabla \cdot v(x)=d\right)=\frac{P\left(\nabla \cdot v(x)=d \mid x \in T_{k}\right) P\left(x \in T_{k}\right)}{P(\nabla \cdot v(x)=d)}
$$

Since the denominator is a normalizing factor, in the following only the numerator is considered. The flux of the subject specific deformations $\exp \left(v_{i}\right)$ through the regions $T_{k}$ can be easily estimated with (1) through a hierarchical model. At the first level, based on spatial priors for the location of the critical points, we can estimate a group-wise confidence map for the critical regions:

- Given a set of critical points $\left\{x_{k}\right\}$, define the spatial priors $P\left(x \in T_{k}\right)=$ $\exp \left(\left(x-x_{k}\right)^{2} /\left(2 \sigma^{2}\right)\right)$

- Define a group-wise prior $\overline{F_{i}^{ \pm}}(x)$ for the critical areas as the group-wise average of the binary masks of positive/negative divergence $F_{i}^{+}=\left\{x \in \Omega \mid \nabla \cdot v_{i}^{T}(x)>0\right\}$, and $F_{i}^{-}=\left\{x \in \Omega \mid \nabla \cdot v_{i}^{T}(x)<0\right\}$.

- From formula (11), define the confidence maps for the critical areas $P_{k}^{ \pm}(x)=$ $P\left(\nabla \cdot v_{i}^{T}(x)=d \mid x \in T_{k}\right) P\left(x \in T_{k}\right)=\overline{F^{ \pm}}(x) \exp \left(\left(x-x_{k}\right)^{2} /\left(2 \sigma^{2}\right)\right.$.

Finally, the group-wise confidence maps are reintroduced in (11) for the second level analysis :

- Transport the confidence maps $P_{k}^{ \pm}$in the subject space to obtain $P_{k, i}^{ \pm}=$ $P_{k}^{ \pm} \circ \psi^{-1}$

- Apply (11) by considering $P\left(x \in T_{k}\right)=P_{k, i}^{ \pm}$, and $F_{i}^{ \pm} \circ \psi_{i}^{-1}$ as likelihood term.

Probabilistic Integration of the Regional Flux. The confidence maps in the subject space can then be used as weights for the integration of the divergence across the space $\Omega$ thanks to the Divergence theorem, to provide a measure of the subject specific flux across the critical regions $T_{k}$. The weighted integration of the divergence implicitly defines the critical regions in a maximum a posteriori approach through the posterior (11), therefore automatically accounting for the registration biases in the anatomical localization (e.g. due to the regularization). 


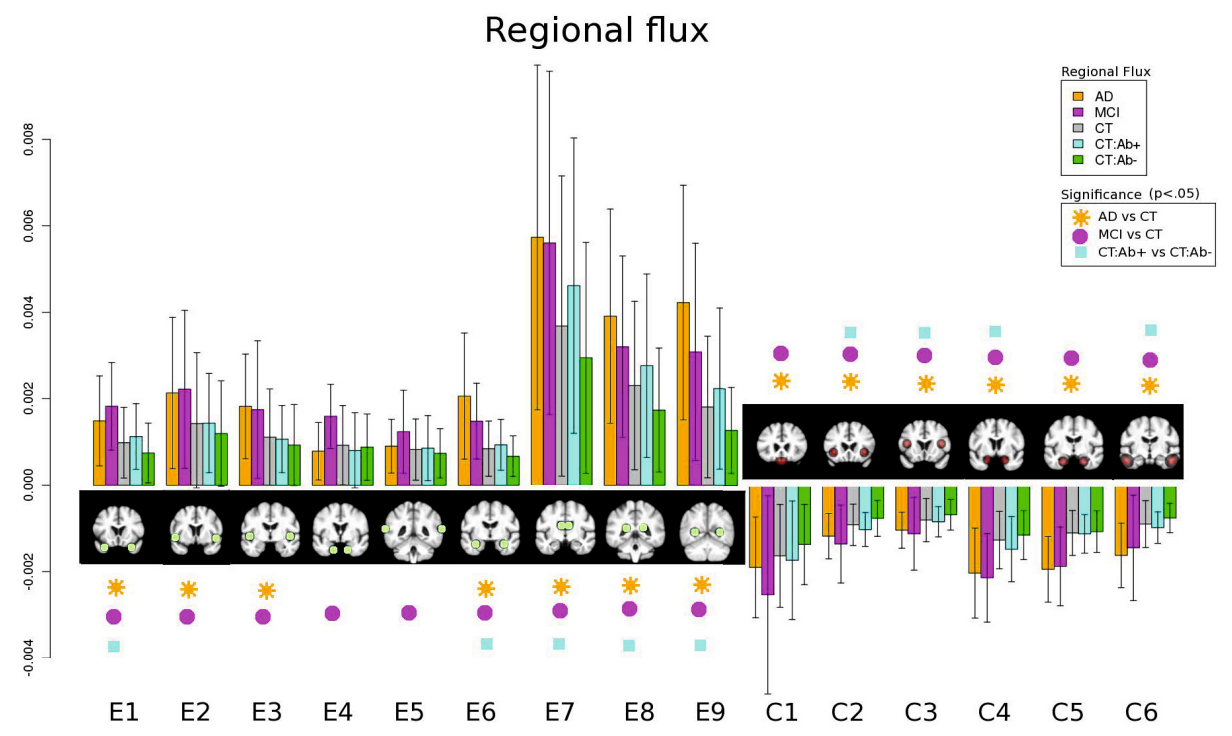

Fig. 3. Average regional flux for AD, MCI, healthy controls, and healthy $A \beta^{+}$and $A \beta^{-}$subgroups. E1 to E9: expanding regions. C1 to C6: contracting regions.

\section{Apparent Gain and Loss of Matter in Alzheimer's Disease through Regional Flux Quantification}

Baseline and one year follow-up brain scans of 200 healthy controls, $150 \mathrm{MCI}$, and $142 \mathrm{AD}$ patients from the ADNI dataset were linearly aligned and non-rigidly registered with the log-Demons. The pressure maps $p_{i}$ corresponding to the intra-subject longitudinal trajectories $\exp \left(v_{i}\right)$ were transported into a previously defined anatomical reference along the subject-to-template deformations $\psi_{i}$.

The set of local maxima and minima for the pressure in $\mathrm{AD}$ has been defined from the mean pressure map associated to the longitudinal deformations of 20 randomly selected $\mathrm{AD}$ patients. Of these sparse sets of points, 9 local minima and 6 local maxima have been manually labeled to define the set $\left\{x_{k}\right\}$ of critical points. The spatial priors $T_{k}$ were defined through inflation (4 voxels neighborhood) and right/left symmetry (Figure 2).

The hierarchical model of Section 3 was used for the regional probabilistic integration of the flux for the remaining patients and the healthy controls. Moreover, the healthy population was stratified depending on the positivity to the CSF $A \beta_{42}$ marker $(<192 \mathrm{pg} / \mathrm{ml}$ ), and the flux analysis was performed to detect the effect of the positivity on the atrophy progression.

Figure 3 summarizes the group-wise regional flux. As we can see, the flux is higher for the ADs and MCIs with respect to the controls. Interestingly, the MCIs have larger flux than the ADs in some regions, which might indicate greater structural longitudinal changes at the early stages of the disease, or underline 
LDA most significant coefficients

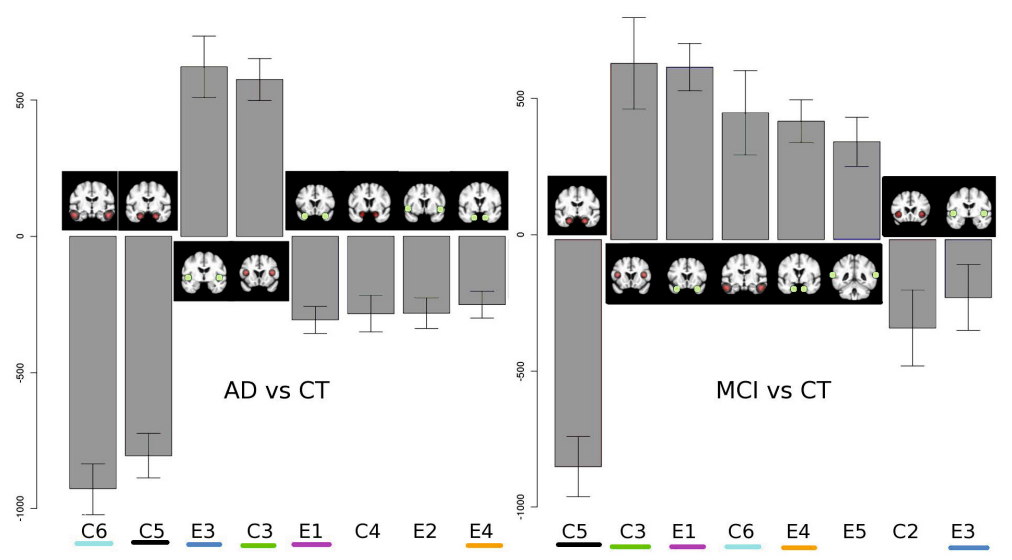

Fig. 4. LDA coefficients associated to the most discriminant critical regions for the longitudinal atrophy of the AD and MCI groups wrt normal aging. Among these regions, 6 of the 8 are common to both $\mathrm{AD}$ and $\mathrm{MCI}$, and are indicated by the common colors.

different aspects of the heterogeneous MCI condition. The subgroup of healthy subject positive to the $A \beta 42$ marker consistently show increased flux when compared to the negative, which is significant for several regions, and might suggest a possible effect of the $A \beta 42$ marker on the future development of AD.

A power analysis based on the regional flux was performed to define the sample size required by an hypothetical 1-year clinical trial to detect a $25 \%$ difference of the progression of the measure with $80 \%$ power when considering the group alone, or by comparing with normal aging [4]. The regional measurements provided different sample size estimations 3 . To summarize, the lowest sample size for the AD group was provided by the flux across C5 (hippocampus): 38 $(95 \%$ CI [33,44]) by considering the AD alone, and $203[145,307]$ when controlling by normal aging. For the MCI group, the lowest sample size was given by the region E4 (mid-temporal pole): 54 [47,63] for the group alone and 307 [192,567] when controlled for normal aging.

Finally a linear discriminant analysis was performed to define the combination of such regions which maximises the flux differences for respectively AD and MCI vs healthy subjects. The analysis was carried out through a 2-folds cross-validation, with 1000 iterations (Figure 4). An additional power analysis was performed during the cross validation, to test the effectiveness of the LDA combination of the regional flux as a clinical measure. The average sample size (and average 95\% CI) required for the LDA score when controlling for normal aging was 164 [106,290] for the AD group, and 277 [166,555] for the MCI.

\footnotetext{
${ }^{3}$ Supplementary material at http://www-sop.inria.fr/members/Marco.Lorenzi/ Flux-MICCAI2012.pdf
} 


\section{Conclusions}

We proposed to decompose the longitudinal trajectories according to the Helmholtz theorem, in order to analyze the atrophy processes through the pressure potential map and the associated flux. This new approach studies the temporal dynamics as a topological problem, and opens the path to new analysis methods based on graph and complex theory. The proposed work provided precise and statistically powered quantifications of the group-wise regional atrophy processes. Moreover the presented method describes and compares the patterns of dynamic changes between clinical populations, and might thus lead to potentially new anatomical findings, such as differential atrophy trajectories at different disease stages.

Acknowledgements. This work was partially funded by the French ANR "programme blanc" number ANR-09-BLAN-0332, and by the European Research Council through the ERC Advanced Grant MedYMA.

\section{References}

1. Fox, N., Crum, W., Scahill, R., Stevens, J., Janssen, J., Rossnor, M.: Imaging of onset and progression of Alzheimer's disease with voxel compression mapping of serial magnetic resonance images. Lancet 358, 201-205 (2001)

2. Thompson, P., Ayashi, K., Zubicaray, G., et al.: Dynamics of gray matter loss in Alzheimer's disease. The Journal of Neuroscience 23, 994-1005 (2003)

3. FreeBorough, P., Fox, N.: The boundary shift integral: An accurate and robust measure of cerebral volume changes from registered repeat MRI. TMI 16(5) (1997)

4. Leung, K.K., Barnes, J., Ridgway, G.R., et al.: Automated cross-sectional and longitudinal hippocampal volume measurement in mild cognitive impairment and Alzheimer's disease. NeuroImage 51(4), 1345-1359 (2010)

5. Hansen, M.S., Larsen, R., Christensen, N.V.: Curl-gradient image warping - introducing deformation potentials for medical image registration using Helmholtz decomposition. In: VISAPP 2009, vol. 1, pp. 179-185 (2009)

6. Chung, Worsley, Paus, et al.: A unified statistical approach to deformation-based morphometry. NeuroImage 14(3), 595-606 (2001)

7. Mueller, S., Weiner, M., Thal, L., et al.: The Alzheimer's disease neuroimaging initiative. Neuroimaging Clin 15, 869-877 (2005)

8. Lorenzi, M., Ayache, N., Frisoni, G.B., Pennec, X.: Mapping the Effects of A $\beta_{1^{-}}$ 42 Levels on the Longitudinal Changes in Healthy Aging: Hierarchical Modeling Based on Stationary Velocity Fields. In: Fichtinger, G., Martel, A., Peters, T. (eds.) MICCAI 2011, Part II. LNCS, vol. 6892, pp. 663-670. Springer, Heidelberg (2011)

9. Vercauteren, T., Pennec, X., Perchant, A., Ayache, N.: Symmetric Log-Domain Diffeomorphic Registration: A Demons-Based Approach. In: Metaxas, D., Axel, L., Fichtinger, G., Székely, G. (eds.) MICCAI 2008, Part I. LNCS, vol. 5241, pp. 754-761. Springer, Heidelberg (2008)

10. Mansi, T., Pennec, X., Sermesant, M., Delingette, H., Ayache, N.: iLogDemons: A Demons-based registration algorithm for tracking incompressible elastic biological tissues. IJCV 9(30), 92-111 (2011) 